\title{
Thermal Variation of Elastic Modulus on Nanocrystalline NiCuZn Ferrites
}

\author{
S. R. Murthy \\ Department of Physics, Osmania University, Hyderabad 500 007, India \\ Correspondence should be addressed to S. R. Murthy; ramanasarabu@gmail.com
}

Received 26 April 2013; Accepted 1 September 2013

Academic Editor: Matjaz Valant

Copyright ( 2013 S. R. Murthy. This is an open access article distributed under the Creative Commons Attribution License, which permits unrestricted use, distribution, and reproduction in any medium, provided the original work is properly cited.

\begin{abstract}
The nanopowders of $\mathrm{Ni}_{0.38} \mathrm{Cu}_{0.12} \mathrm{Zn}_{0.5} \mathrm{Fe}_{2} \mathrm{O}_{4}$ with particle size, $20 \mathrm{~nm}$ have been synthesised using Microwave-Hydrothermal method and characterized. Then the ferrite samples were microwave sintered at different temperatures in an air atmosphere and characterized. The magnetic properties were measured at room temperature. The dielectric constant $(\varepsilon)$, initial permeability $\left(\mu_{i}\right)$ and quality factor $(Q)$ has been measured on sintered samples at $1 \mathrm{MHz}$. Thermal variation of initial permeability has been measured over temperature range of $300 \mathrm{~K}-600 \mathrm{~K}$. A detailed study of elastic behaviour of NiCuZn ferrites has been under taken using a composite piezoelectric oscillator method over a temperature of $300 \mathrm{~K}-600 \mathrm{~K}$. The room temperature elastic moduli is found to be slightly sample dependent and decreases with increasing the temperature, except near the Curie temperature, $T_{C}$, where a small anomaly is observed. The internal friction at room temperature is also found to be more particle size dependent. The temperature variation of internal friction exhibits a broad maximum around $500 \mathrm{~K}$, just below Curie temperature $T_{C} 530 \mathrm{~K}$. The above observations were carried on in the demagnetized state; on the application of a $400 \mathrm{mT}$ magnetic field allowed us to reach the saturated state of the sample at any of the measuring temperature. The anomaly observed in the thermal variation of elastic moduli and internal friction is explained with the help of temperature variation of magneto-crystalline anisotropy constant.
\end{abstract}

\section{Introduction}

By the development of surface mount technology (SMT) and multilayer chip devices, NiCuZn ferrites have been extensively studied and widely used to fabricate chip inductors and EMI filters because of their relatively low sintering temperature and high resistivity with good performance at high frequencies [1-5]. However, in these ferrites, it was found that the mechanical and magnetic properties are easily changed or deteriorated by the stress caused at the internal electrode. These problems can be reduced by the preparation of $\mathrm{NiCuZn}$ ferrites under controlled experimental conditions and with the knowledge of mechanical properties. On the other hand, the mechanical properties of ferrites are often taken for granted during the manufacture and testing. Despite this, the strength of ferrites can be important particularly for parts, which are tightly clamped during their assembly and use to assure minimum changes in devices performance with mechanical shock, temperature, vibration, and so forth. A survey of the literature tells that no information is available for $\mathrm{NiCuZn}$ ferrites on the elastic properties.
There is an abundant literature on the propagation of ultrasonic waves in magnetic materials, but a few interpretations only refer to possible effects due to the domain wall (DW) [6]. It was known from the acoustic emission (AE) studies on magnetic materials that the space occupied by a DW is the location of magnetoelastic interactions and ultrasonic bursts are emitted when the DW are created or annihilated $[7,8]$. It was also found that the specific DW/lattice magneto-elastic interactions might be one of the origin of the DW relaxation. In order to understand, an interaction of DW with elastic modulus, a detailed study of the thermal variation of elastic modulus, and internal friction on nanocrystalline $\mathrm{Ni}_{0.38} \mathrm{Cu}_{0.12} \mathrm{Zn}_{0.5} \mathrm{Fe}_{2} \mathrm{O}_{4}$ have been undertaken over a wide temperature range.

\section{Experimental Method}

Pure (99.98\%) nickel nitrate $\left[\mathrm{Ni}\left(\mathrm{NO}_{3}\right) 2.6 \mathrm{H}_{2} \mathrm{O}\right],(99.95 \%)$ copper nitrate $\left[\mathrm{Cu}\left(\mathrm{NO}_{3}\right) 2.3 \mathrm{H}_{2} \mathrm{O}\right],(99.98 \%)$ zinc nitrate 
$\left[\mathrm{Zn}\left(\mathrm{NO}_{3}\right) 2.6 \mathrm{H}_{2} \mathrm{O}\right]$, and $(99.96 \%)$ iron nitrate $\left[\mathrm{Fe}\left(\mathrm{NO}_{3}\right)\right.$ $2.9 \mathrm{H}_{2} \mathrm{O}$ ] were dissolved in $50 \mathrm{~mL}$ of deionized water. The molar ratio of powders was adjusted to obtain the composition $\mathrm{Ni}_{0.38} \mathrm{Cu}_{0.12} \mathrm{Zn}_{0.5} \mathrm{Fe}_{2} \mathrm{O}_{4}$. An aqueous $\mathrm{NaOH}$ solution was added to solution until the desired $\mathrm{pH}(\sim 9.45)$ value was obtained. The mixture was then treated in Teflon lined vessel using a microwave digestion system (Model MDS2000, CEM Corp., Mathews, NC). This system uses $2.45 \mathrm{GHz}$ microwaves and can operate at $0-100 \%$ full power $(630 \pm$ $50 \mathrm{~W}$ ). In the present investigation, the samples were synthesized at $160^{\circ} \mathrm{C} / 30 \mathrm{~min}$. The time, pressure, and power were computer controlled. The products obtained were filtered and then washed repeatedly with deionized water, followed by freeze drying overnight. The synthesized powders were weighed, and the percentage yields were calculated from the expected and the amount that was actually crystallized. The yield obtained in the present investigation was $95 \%$. As synthesized powders were characterized by using powder Xray diffraction (XRD, Philips analytical X-ray diffractometer with $\mathrm{Cu}-\mathrm{K} \alpha$ radiation). Particle size and morphology were determined using transmission electron microscopy (TEM, Model JEM-2010, JEOL, Tokyo, Japan).

To nanopowders, $2 \mathrm{wt} \%$ of poly-vinyl-alcohol was added as a binder and pressed in a die under a pressure of $190 \mathrm{MPa}$ for $10 \mathrm{~min}$ into pellets $(10 \mathrm{~mm}$ diameter, $3 \mathrm{~mm}$ thickness), and (50 $\mathrm{mm}$ in length, $10 \mathrm{~mm}$ diameter), and toroids $(12 \mathrm{~mm}$ outside diameter, $6 \mathrm{~mm}$ inside diameter, $3 \mathrm{~mm}$ thickness). After binder burnt out at $300^{\circ} \mathrm{C}$, the samples were microwave sintered at $750^{\circ} \mathrm{C} / 30 \mathrm{~min}$ (MW1), $800^{\circ} \mathrm{C} / 30 \mathrm{~min}(\mathrm{MW} 2), 850^{\circ} \mathrm{C} / 30 \mathrm{~min}(\mathrm{MW} 3), 900^{\circ} \mathrm{C} / 30 \mathrm{~min}$ (MW4), and $930^{\circ} \mathrm{C} / 30 \mathrm{~min}$ (MW5) in air. The microwave sintering process was carried out using a specially designed applicator which consists of a domestic microwave oven having an output power level tuneable up to a maximum of $800 \mathrm{~W}$ and operating frequency of $2.45 \mathrm{GHz}$.

All the sintered samples were characterized using the $\mathrm{X}$-ray diffraction (XRD). The average grain size (Dm) of the samples was evaluated using the scanning electron microscopy (SEM; LEICA, S440i, UK) pictures. The thermal expansion of the sintered samples has been measured using dilatometer (DIL 402C) in air and at a heating rate of $20^{\circ} \mathrm{C} / \mathrm{min}$. The saturation magnetization $\left(M_{S}\right)$ and coercive field $\left(H_{C}\right)$ values of the present samples were obtained from the recorded hysteresis loops using the vibrating sample magnetometer (VSM, model DMS 1660) at room temperature. The initial permeability $\left(\mu_{i}\right)$ and quality factor has been measured on sintered samples at $1 \mathrm{MHz}$ using a LCR meter. Thermal variation of initial permeability has been measured using the LCR meter at $1 \mathrm{kHz}$.

The elastic constants such as Young's modulus, $Y$, and shear modulus, $G$, were measured on sintered samples over the temperature range of $300 \mathrm{~K}-600 \mathrm{~K}$ using the composite piezoelectric oscillator method [9]. The appropriate quartz crystal transducer was cemented on the specimen with phenyl salicylate and two parts of sodium silicofluoride mixed with one part of barium sulphate in one drop of water glass for room and high temperature measurements, respectively. For Young's moduli measurements, the piezoelectric crystals were cut to oscillate at $100.245 \pm 0.001 \mathrm{kHz}$ in longitudinal direction. Shear moduli were measured on the same specimen sections, using shear crystals oscillating at approximately $165.25 \pm 0.002 \mathrm{kHz}$. Young's and shear moduli were then determined in the usual way using the following expressions: $Y=\left(\lambda_{l} f_{l}\right)^{2} \rho, G=\left(\lambda_{s} f_{s}\right)^{2} \rho$, where $\rho$ is density, $\lambda$ is the wavelength, $f$ is the resonant frequency, and subscripts "l" and "s" refer to longitudinal and shear waves, respectively. All the measurements were conducted keeping the composite oscillator in a vacuum $\left(10^{-4}\right.$ Torr $)$. The vacuum chamber was placed at the centre of pole pieces of an electromagnet. The magnetic field was always applied parallel to the length of the specimen. The temperature of the sample is measured using $\mathrm{Cr}$-Al thermocouple with an accuracy of $\pm 0.1 \mathrm{~K}$. The accuracy of $Y, G$, and internal friction measurements is about $0.1 \%$, $0.1 \%$, and $0.5 \%$ respectively.

\section{Results and Discussion}

Figure 1(a) gives the XRD patterns for as-synthesized powder of NiCuZn ferrite. It can be seen from the figure that the phase-pure ferrite was obtained. The broad peaks in the XRD pattern indicate the formation of nanopowders. All the peaks in the diffraction pattern have been indexed. The peaks (220), (311), (400), (511), and (440) have been deconvoluted to Lorentzian curves using Peak Fit software for the determination of the average diameter (DXRD) using full-width at half-maximum (FWHM) value. The average particle size $\left(D_{m}\right)$ has been calculated using the Scherrer formula: $D_{m}=k l / b \cos \theta$, where $k$ is a constant, $b$ is the full width half maxima, $l$ is the wavelength of X-rays used, and $\theta$ is the diffraction angle. The average particle size of $\mathrm{NiCuZn}$ ferrite powder was found to be $\sim 25 \mathrm{~nm}$.

Figure 1(b) shows TEM picture for the as-synthesized ferrite powder. The particles are nearly spherical in shape. The average particle size calculated from the different regions of the image was $20 \mathrm{~nm}$. The selected area electron diffraction (SAED) pattern shows rings made up of discrete spots, indicating high crystallinity; the rings are consistent with the cubic spinel structure with an intense ring pattern form $(h k l)$ planes. No secondary phases are found. The observed average size is consistent with that obtained from the $\mathrm{X}$-ray analysis.

The powder XRD patterns for microwave sintered NiCuZn ferrites under investigation reveal a single-phase cubic spinel structure without any other impurity phases (Figure 2). The lines are board, which is a typical characteristic of nanocrystalline nature. The grain sizes were calculated from the XRD line broadening of the peak (311) using the Scherrer relationship, and the obtained values are presented in Table 1. Figure 3 shows the typical SEM pictures for microwave sintered samples. It can be seen from the figures that with an increase of sintering temperature, microstructure of the ferrite has become finer and finer. The microwave sintered samples exhibit fine and uniform microstructures due to the small soaking time. The grain sizes $\left(D_{m}\right)$ as measured from SEM picture for the presently investigated ferrites were presented in Table 1. It can be observed from the table that the grain size of sintered ferrites increases with an increase of sintering temperature. 


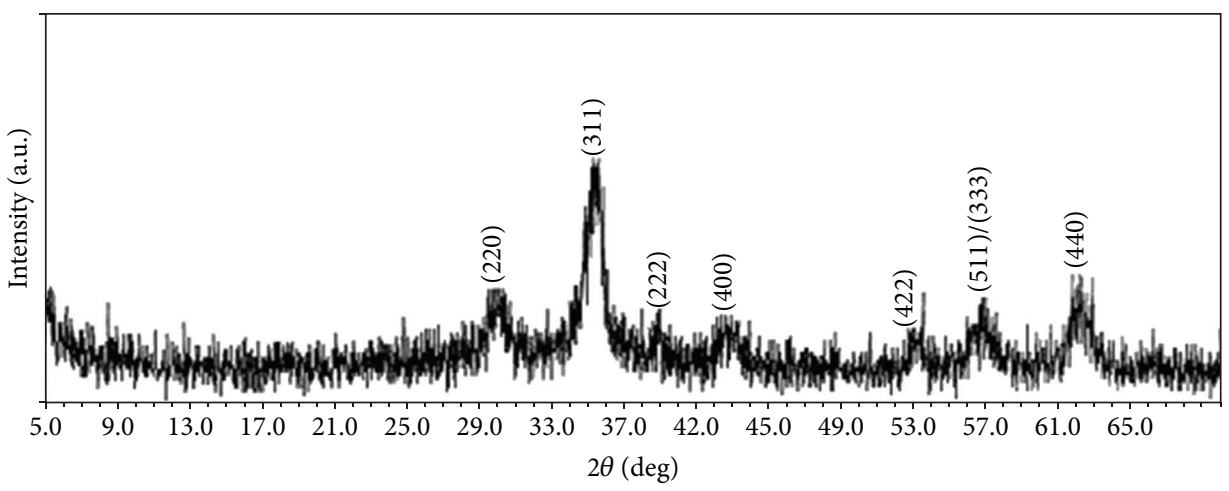

(a)
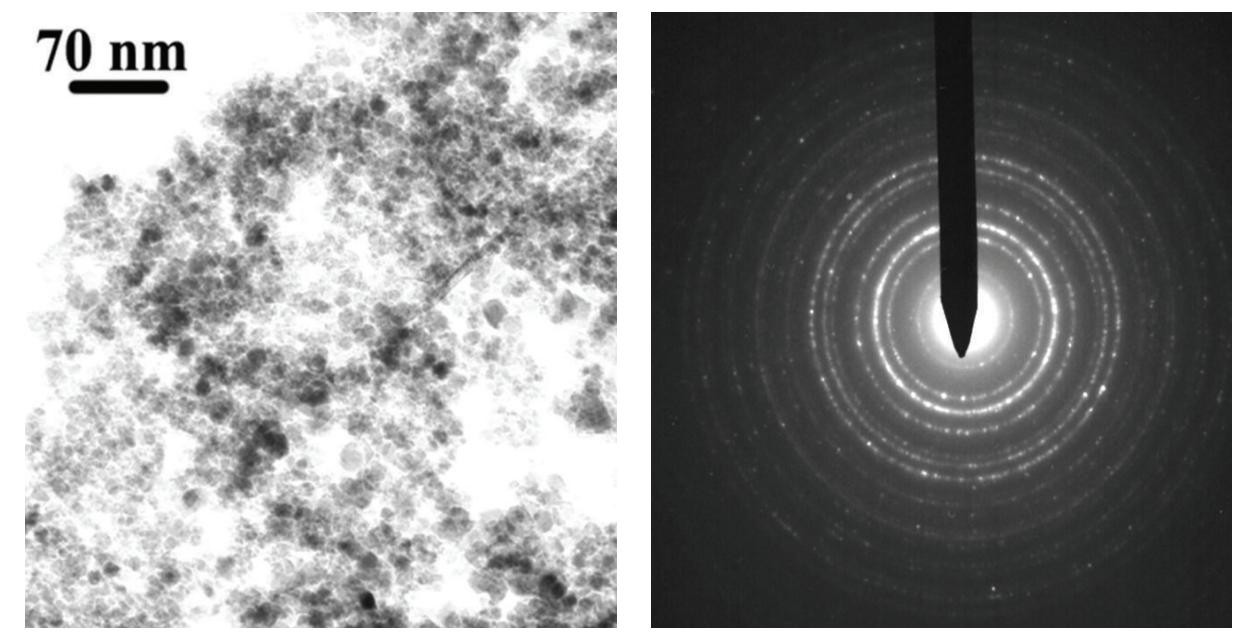

(b)

FIgURE 1: (a) XRD pattern and (b) TEM picture for as-synthesized ferrite powder.

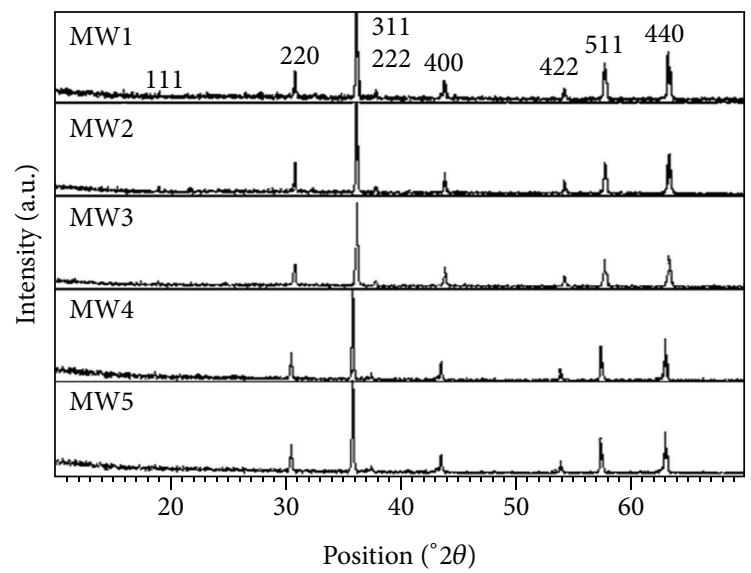

FIGURE 2: XRD patterns for microwave sintered NiCuZn ferrites.

The value of lattice constant $(a)$ has been calculated using the XRD data, and the average value of lattice constant is $8.3374 \pm 0.0001 \AA$. The bulk density $\left(d_{X}\right)$ of the sintered samples has been measured using Archimedes's method, and the obtained results are presented in Table 1. It can be observed from the table that the value of bulk density increases with an increase of sintering temperature. It can also be seen from the table that the theoretical density (TD) samples have reached $97 \%$ at a sintering temperature of $750^{\circ} \mathrm{C}$. Finally, a theoretical density of $99.5 \%$ has been achieved at a low sintering temperature of $930^{\circ} \mathrm{C}$. The ultrafine particle size and relatively uniform grains are 


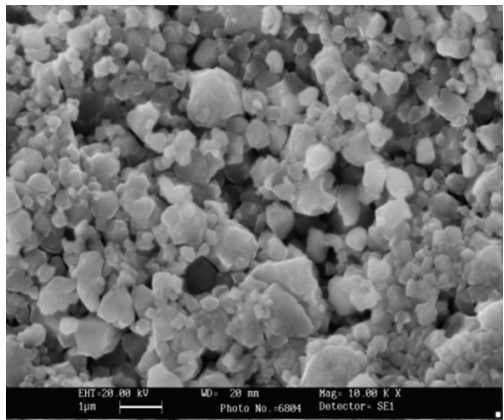

(a)

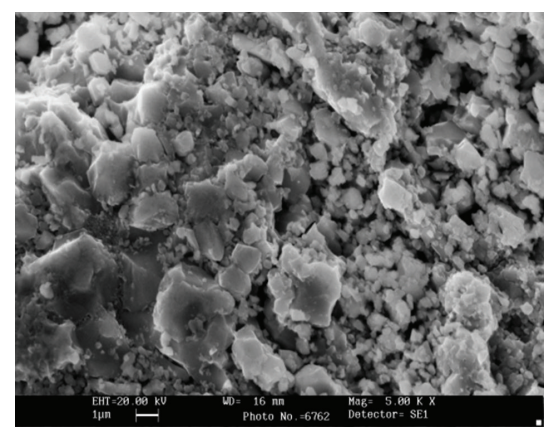

(b)

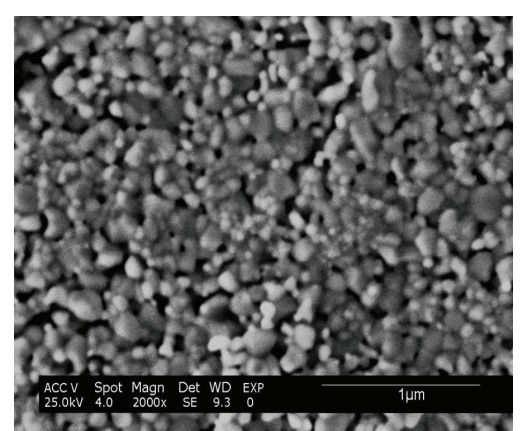

(c)

FIGURE 3: SEM pictures for microwave sintered samples (a) MW3, (b) MW4, and (c) MW5.

TABLE 1: Room temperature data on NICuZn ferrites.

\begin{tabular}{lccccccccc}
\hline Sample & $T_{S}\left({ }^{\circ} \mathrm{C} / \mathrm{min}\right)$ & $d_{X}(\mathrm{~g} / \mathrm{cc})$ & $\mathrm{TD} \%$ & $D_{m}(\mathrm{~nm})$ & $\begin{array}{c}\varepsilon \\
1 \mathrm{MHz}\end{array}$ & $\begin{array}{c}\mu_{i} \\
1 \mathrm{MHz}\end{array}$ & $\begin{array}{c}Q \\
1 \mathrm{MHz}\end{array}$ & $M_{S}(\mathrm{emu} / \mathrm{g})$ & $H_{C}(\mathrm{Oe})$ \\
\hline MW1 & $750 / 30$ & 5.312 & 97 & 80 & 12 & 448 & 50 & 50 \\
MW2 & $800 / 30$ & 5.325 & 97.1 & 68 & 12 & 460 & 50 & 53 \\
MW3 & $850 / 30$ & 5.376 & 98 & 56 & 10 & 485 & 53 & 60 \\
MW4 & $900 / 30$ & 5.398 & 98.4 & 45 & 10 & 510 & 60 & 68 \\
MW5 & $930 / 30$ & 5.459 & 99.5 & 35 & 8 & 535 & 65 & 76 \\
\hline
\end{tabular}

TABLE 2: Elastic modulus data for NiCuZn ferrites at room temperature.

\begin{tabular}{lccccccc}
\hline Sample name & $Y \times 10^{-10}\left(\mathrm{~N} / \mathrm{m}^{2}\right)$ & $G \times 10^{-10}\left(\mathrm{~N} / \mathrm{m}^{2}\right)$ & $K \times 10^{-10}\left(\mathrm{~N} / \mathrm{m}^{2}\right)$ & $Q^{-1}$ & $V_{l}(\mathrm{~m} / \mathrm{s})$ & $V_{S}(\mathrm{~m} / \mathrm{s})$ & $\alpha$ \\
\hline MW1 & 17.82 & 7.28 & 10.76 & 0.71 & 6345 & 3345 \\
MW2 & 18.03 & 7.34 & 11.12 & 0.65 & 6468 & 3675 \\
MW3 & 18.10 & 7.45 & 8.42 & 0.45 & 6675 & 3890 \\
MW4 & 18.25 & 7.56 & 10.39 & 0.26 & 6915 & 3962 \\
MW5 & 18.54 & 7.67 & 9.38 & 0.20 & 6920 & 3974 \\
\hline
\end{tabular}

responsible for achieving this very high densification at low temperature. We could not reach theoretical value $(99.9 \%$ of TD) because of the ferrite particle being too fine to be completely dispersed in the liquid suspension and, therefore, flocculated to some extent by Van der Waals bonding. This slightly flocculated suspension resulted in some agglomerated regions distributed in the microstructure of samples. This was confirmed by microstructure. Rearrangement and differential micro-densification processes could not, thus, be wholly avoided during the sintering of these slightly inhomogeneous packed compacts, and therefore completely dense fired bodies were not achieved.

Magnetic properties such as saturation magnetization $\left(M_{S}\right)$ and coercivity $\left(H_{C}\right)$ obtained from the recorded hysteresis loops are presented in Table 1 . The NiCuZn ferrite sintered at $930^{\circ} \mathrm{C} / 30 \mathrm{~min}$ exhibits optimum magnetic property, a saturation magnetization of $76 \mathrm{emu} / \mathrm{g}$, and $H_{C}=60 \mathrm{Oe}$. It is also observed from the table that value of $M_{S}$ increases with increasing sintering temperature, as a result of increasing crystallinity. The values of $H_{C}$ decrease with increasing sintering temperature. From the magnetic properties results, one can notice that the maximum $M_{S}$ corresponds to a smaller particle size of $35 \mathrm{~nm}$; thus, control of crystal growth by varying sintering temperature can produce ultrafine magnetic powders.

Table 1 also gives initial permeability $\left(\mu_{i}\right)$, dielectric constant $(\varepsilon)$, and quality factor $(Q)$ values for sintered ferrites at $1 \mathrm{MHz}$. It can be seen from the table that the samples sintered at $930^{\circ} \mathrm{C} / 30 \mathrm{~min}$ exhibit an optimum $\mu_{i}$ value of 535. It can be observed from the table that $\mu_{i}$ increases with increasing sintering temperature from $750^{\circ} \mathrm{C}$ to $930^{\circ} \mathrm{C}$ as a result of increasing densification. The ferrite sintered at $930^{\circ} / 30 \mathrm{~min}$. also shows a maximum quality factor value of 65 . The dielectric constant for present ferrites varies from 8 to 12 . The smaller dielectric constant has been observed for MW5 sample.

The elastic constants such as Young's $(Y)$, shear modulus $(G)$, and internal friction $\left(Q^{-1}\right)$ were measured at room temperature on sintered samples and presented in Table 2. The table also gives the computed values of Bulk modulus $(B=(Y G) / 3(3 G-Y)$. It can be seen from table that the values of $Y$ and $G$ are found to increase with an increase 
of sintering temperature. This shows that an increase of sintering temperature increases the atomic binding force markedly. The average value of Poisson's ratio $(\sigma=(Y / 2 G)-$ 1 ) for the samples under investigation is 0.3 . The microwave sintered ferrites possess higher values of $Y$ and $G$ when compared to those of ferrites prepared by using solid state sintering method [10]. This is due to the high densification of the present samples.

It is clear from the table that the room temperature $Y$ and $G$ were found to increase from $17.82 \mathrm{~N} / \mathrm{m}^{2}$ to $18.54 \mathrm{~N} / \mathrm{m}^{2}$ and from $7.28 \mathrm{~N} / \mathrm{m}^{2}$ to $7.67 \mathrm{~N} / \mathrm{m}^{2}$ with an decreasing the grain size from $80 \mathrm{~nm}$ to $35 \mathrm{~nm}$, respectively. Reciprocally, the internal friction was found to decrease from 0.71 to 0.20 . In the present samples, the increase in density has decreased the grain size from $80 \mathrm{~nm}$ to $35 \mathrm{~nm}$. As the porosity $(\sim 0.3)$ of samples remained nearly constant, the increase in the elastic modulus and decrease in the internal friction may be considered due to an decrease in the grain size. This is because, presently, microwave sintered samples have densities in the range from $5.312 \mathrm{~g} / \mathrm{cm}^{3}$ to $5.459 \mathrm{~g} / \mathrm{cm}^{3}$. This density variation is small compared to the variation of grain size $(35 \mathrm{~nm}$ to $80 \mathrm{~nm})$. Therefore grain size, not density, has the dominant effect on elastic properties.

No elastic modulus data is available in the literature on nanocrystalline NiCuZn ferrites. However, the present elastic modulus data is in fair agreement with that of previously investigated elastic behaviour of several polycrystalline ferrites [11-13]. Using the elastic modulus data, we have estimated the longitudinal velocity $\left(V_{l}\right)$ and shear velocity $\left(V_{s}\right)$ for all the samples, and results are presented in Table 2. The values of $V_{l}$ and $V_{s}$ are found to increase with an increase of sintering temperature. With the help of velocities, the Debye characteristic temperature $(\Theta)$ has been estimated using the Anderson method [14]. The average value of the Debye temperature for present ferrites is $546 \mathrm{~K}$.

The elastic behaviour on $\mathrm{NiCuZn}$ ferrites has been measured in the temperature range of $300 \mathrm{~K}-600 \mathrm{~K}$. For these experiments, a fused silica buffer rod was used to isolate the piezoelectric crystals from the heated specimen [15]. Fractional variation in frequency was measured and related to fractional modulus variations through the expansion coefficient.

Consider that $Y_{T}$ or $G_{T} / Y_{R T}$ or $G_{R T}=(1+\alpha \Delta T)^{-1}$ $\left(f_{s T} / f_{s}\right)^{2}$, where $Y_{R T}, G_{R T}$, and $f_{s}$ are Young's modulus, shear modulus, and resonance of the specimen at the reference temperature (room temperature or $300 \mathrm{~K}$ ), $Y_{T}, G_{T}$, and $f_{s T}$ are the same quantities at any other temperature, $\Delta T$ is the difference between the measured and reference temperature, and $\alpha$ is the coefficient of linear expansion over the measured temperature range.

The values of the coefficient of thermal expansion $(\alpha)$ for the present specimens are measured using the dilatometer method in the temperature range of $300 \mathrm{~K}-600 \mathrm{~K}$. It was found that the variation of " $\alpha$ " with temperature $(T)$ for samples MW1, MW2, MW3, MW4, and MW5 are given by the following:

$$
\alpha=6.261 \times 10^{-6}+0.65 \times 10^{-8}(T)+0.532 \times 10^{-11}\left(T^{2}\right),
$$

$$
\alpha=6.372 \times 10^{-6}+0.75 \times 10^{-8}(T)+0.58 \times 10^{-11}\left(T^{2}\right)
$$

$$
\alpha=6.485 \times 10^{-6}+0.92 \times 10^{-8}(T)+0.542 \times 10^{-11}\left(T^{2}\right),
$$

$$
\alpha=6.578 \times 10^{-6}+0.71 \times 10^{-8}(T)+0.645 \times 10^{-11}\left(T^{2}\right),
$$

$$
\alpha=6.278 \times 10^{-6}+0.25 \times 10^{-8}(T)+0.141 \times 10^{-11}\left(T^{2}\right) .
$$

Figures 4 and 5 give the thermal variation of Young's and shear modulus measured in the demagnetized state, for all samples. It can be seen from the figures that the values of $Y$ and $G$ decrease smoothly with increasing temperature and attain a minimum at a certain temperature $\left(T_{1}\right)$ below the Curie point. Beyond this temperature the elastic modulus shows a positive temperature coefficient and attains a maximum value at a temperature $T_{2}$. With further increase of temperature the both $Y$ and, $G$ are found to decrease. For samples, MW2, MW3, and MW4, the values of $Y$ are very close to each other and are found to decrease by about $14 \%$ between $300 \mathrm{~K}$ and $T_{C}$. In all these samples, anomalous behaviour occurs in between $520 \mathrm{~K}$ and $530 \mathrm{~K}$. The thermal variation of $G$ for all the samples is found to be similar and the variation is about $5 \%$ between $300 \mathrm{~K}$ and $T_{C}$. A less $(525 \mathrm{~K}$ to $535 \mathrm{~K}$ ) pronounced increase of $G$ with temperature is observed near the Curie point. No hysteresis has been observed during cooling. At a given temperature elastic modulus decreases when the mean grain size increases; this tendency is maintained all over the investigated temperature range. The decrease of $Y$ and $G$ with temperature is mainly due to the softening of the material. Murthy et al. $[12,13,16,17]$ also observed a similar variation of $V_{l}$ and $V_{s}$ with temperature in the vicinity of Curie temperature in case of polycrystalline YIG, and observed changes were used to understand the interaction of ultrasonic waves with annihilation/creation of domain walls.

The anomalous behaviour observed in the case of the presently investigated ferrites can be explained qualitatively with help of the temperature variation of the magnetocrystalline anisotropy constant $\left(k_{1}\right)$. The temperature variation of $k_{1}$ was measured on the brother samples and found that $k_{1}$ becomes zero just below the Curie temperature, that is, at $522 \pm 1 \mathrm{~K}$. The measurements of initial permeability versus temperature on the brother sample have shown that the Curie temperature of the present samples is $530 \pm 0.1 \mathrm{~K}$.

As can be seen from Figures 4 and 5 , the $Y$ and $G$ for present samples have reached a minimum value at temperature $520 \mathrm{~K}$ and $525 \mathrm{~K}\left(T_{1}\right)$, while $k_{1}$ becomes zero at $522 \mathrm{~K}$. Similarly, the $Y$ and $G$ attain a maximum value at a temperatures $530 \mathrm{~K}$ and $535 \mathrm{~K}\left(T_{2}\right)$, respectively, while the Curie point of samples under investigation is $530 \mathrm{~K}$. Thus, the minimum $\left(T_{1}\right)$ and maximum $\left(T_{2}\right)$ temperatures of $Y$ and $G$ verses temperature plots coincide with the temperatures of $k_{1}=0$ and the Curie temperature, respectively.

The magneto-crystalline anisotropy constant can be considered as a measure of the magnetic energy barrier to the movement of domain walls in the magnetic materials. 


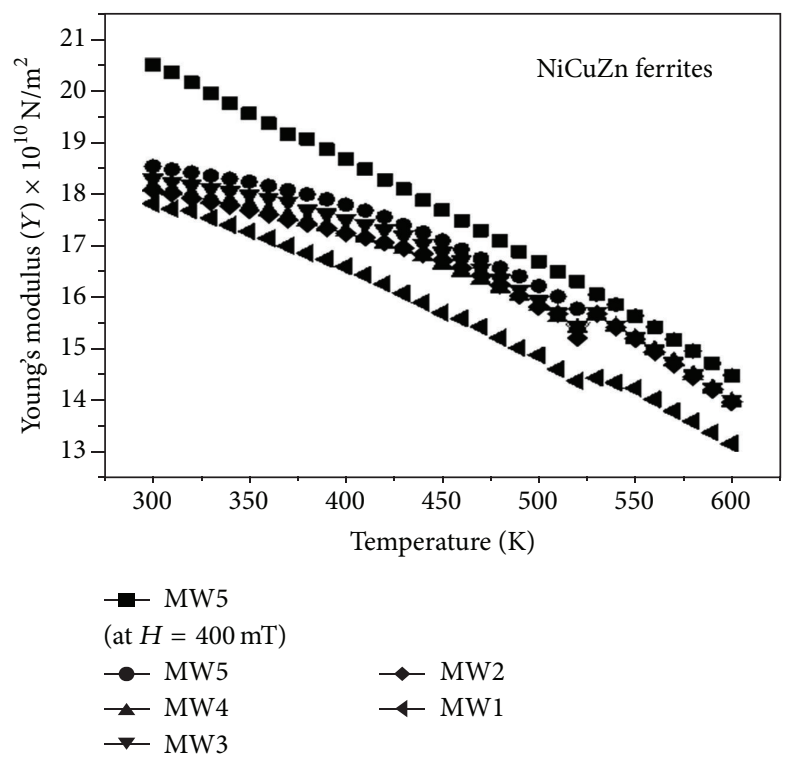

FIGURE 4: Thermal variation of Young's modulus $(Y)$ with temperature for microwave sintered $\mathrm{NiCuZn}$ ferrites.

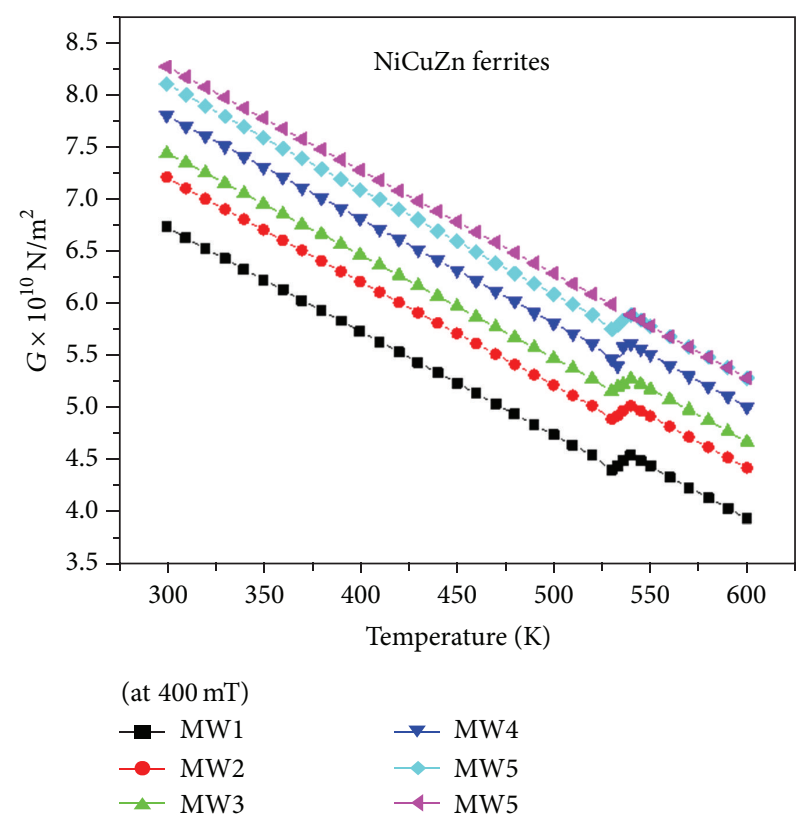

FIGURE 5: Thermal variation of shear modulus $(G)$ with temperature for microwave sintered $\mathrm{NiCuZn}$ ferrites.

As such, domains will be free to move at a temperature at which $k_{1}=0$; the substance undergoes a maximum strain for a given stress; in other words, the $Y$ and $G$ increases till the Curie temperature is reached. Beyond the Curie temperature, both the elastic moduli have shown a decrease with an increase of temperature, since at $T_{C}$ the ferrite losses its spontaneous magnetization and becomes paramagnetic.

In Figures 4 and 5 for the sample MW5, we present the temperature dependence of $Y$ and $G$ measured with $H=$ $400 \mathrm{mT}$; this field was checked to be high enough to saturate

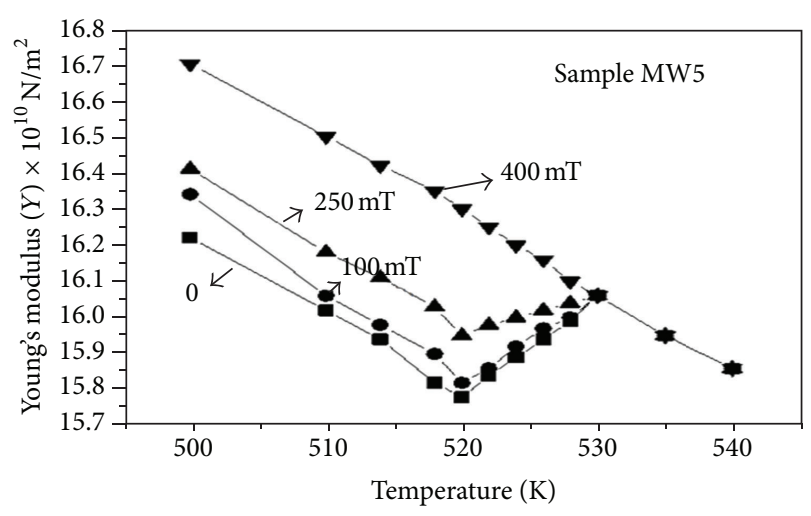

Figure 6: Thermal variation of Young's modulus $(Y)$ with temperature for microwave sintered $\mathrm{NiCuZn}$ ferrites in the vicinity of the Curie temperature at applied magnetic fields.

the sample at any of the measuring temperatures. In saturated state $Y$ (or $G$ ) is slightly higher than in the demagnetized state, expect above $T_{C}$, where both the values are identical, as expected. When the sample is subjected to magnetic field, it is observed that the minimum of elastic moduli-temperature curve is shifted to the low temperature side and becomes shallow. As the magnetic field increased further the minimum continuous to shift to the low temperature side becoming more and more shallow. Finally, the dip disappears when the magnetic field equal to saturation field of the sample (Figure 6).

The magnetic contribution to the velocity variations with temperature can be considered as a "second order effect," superimposed on the purely mechanical effects; it is more pronounced for the large grain size samples. The magnetic contribution $d Y$ defined as the difference between the elastic modulus in the saturated and demagnetized states is, respectively, given by $d Y_{S}=Y_{S}(H=400 \mathrm{mT})-Y(H=0)$ which is not more than $2 \%$ of the total elastic moduli and varies like the temperature variation of saturation magnetization $\left(M_{S}\right)$. Similar variation of $Y$ on temperature and magnetic field was observed for all the other samples.

Figure 7 gives the thermal variation of internal friction $\left(Q^{-1}\right)$ measured at zero fields for all samples. It can be seen from the figure that the sample shows a small maximum around $400 \mathrm{~K}$ and broad maximum just below the Curie temperature. The rapid increase of $Q^{-1}$ has been extended over $10 \mathrm{~K}$ below $T_{C}$ : the very sharp decrease of $Q^{-1}$ from about 0.97 down to 0.2 is accomplished in less than $\pm 2 \mathrm{~K}$. This abrupt change is the signature of the high chemical homogeneity of our samples.

In Figure 7, for the sample MW5, we present the temperature dependence of $Q^{-1}$ measured with $H=400 \mathrm{mT}$. The value of $Q^{-1}$ in the saturated state is almost temperature independent $(=0.17)$ and is equal to the zero field value above $T_{C}$. Similar variation of $Q^{-1}$ on temperature and magnetic field was also observed for all the other samples under investigation, it is observed that the temperature corresponding to the peak in the $Q^{-1}$ versus temperature curve is near the temperature at which both the velocities 


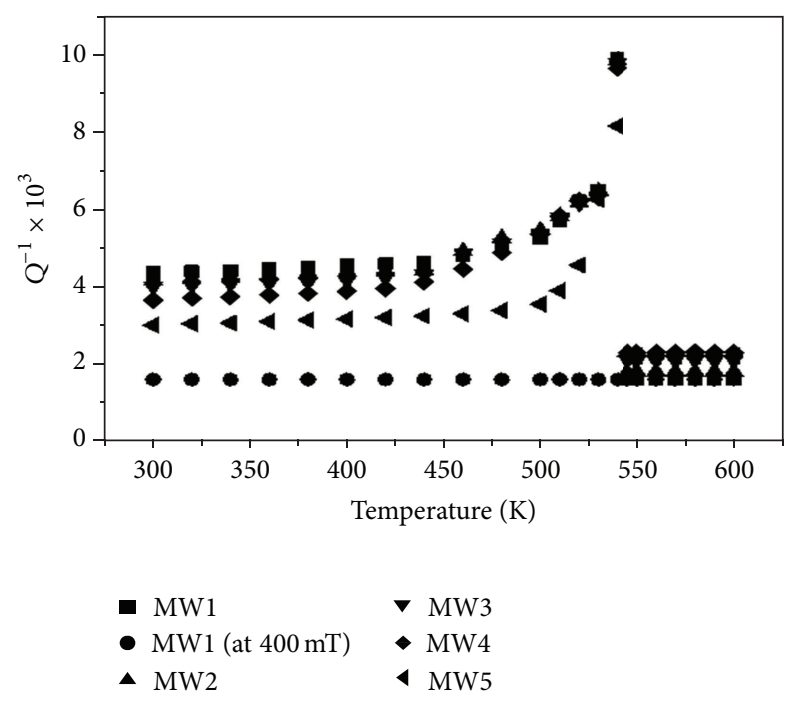

FIgURE 7: Thermal variation of internal friction $\left(Q^{-1}\right)$ with temperature for microwave sintered $\mathrm{NiCuZn}$ ferrites.

show maximum value. As the internal friction is the complementary phenomena of the elastic behaviour, as such the peak observed in $Q^{-1}$ versus $T$ curve can be attributed to the $k_{1}=0$. As the internal friction is the complementary phenomena of elastic behaviour, the qualitative explanation given above can be extended for the occurrence of peaks in the $Q^{-1}$ versus $T$ curves.

The effects of microstructure are well marked on the internal friction, but they are not as dominant as for the elastic modulus. It can be seen from Figure 7 that the microstructure contribution to $Q^{-1}$ is of the order of $95 \%$ of the total. The substantial magnetic contribution to $Q^{-1}$ seems to result from two contributions: an electronic diffusion related magnetoelastic interaction in the $400 \mathrm{~K}$ regions and an interaction of ultrasonic waves with the domain walls in the vicinity of $T_{C}$. We know that the ferrites exhibit magnetic after-effects due to small deviations from the stoechiometry and the relaxation time of which falls in the microsecond range above room temperature; consequently, an ultrasonic wave can induce - through the magnetoelastic couplingspin rotations or domain wall movements, which one leads to electronic migrations: this can explain the relative maximum of $Q^{-1}$ observed around $400 \mathrm{~K}$.

\section{Acknowledgment}

The author is thankful to UGC-BSR, New Delhi, for providing grants to carryout this work.

\section{References}

[1] R. Lebourgeois, J. Ageron, H. Vincent, and J. P. Ganne, "Low losses NiZnCu ferrites," in Proceedings of the 8th International Conference on Ferrites (ICF '00), Tokyo, Japan, 2000.

[2] S. Yan, J. Geng, L. Yin, and E. Zhou, "Preparation of nanocrystalline $\mathrm{NiZnCu}$ ferrite particles by sol-gel method and their magnetic properties," Journal of Magnetism and Magnetic Materials, vol. 277, no. 1-2, pp. 84-89, 2004.

[3] Y.-P. Fu, C.-H. Lin, and C.-W. Liu, "Preparation and magnetic properties of $\mathrm{Ni}_{0.25} \mathrm{Cu}_{0.25} \mathrm{Zn}_{0.5}$ ferrite from microwave-induced combustion," Journal of Magnetism and Magnetic Materials, vol. 283, no. 1, pp. 59-64, 2004.

[4] H. Su, H. Zhang, and X. Tang, "Effects of $\mathrm{Bi}_{2} \mathrm{O}_{3}-\mathrm{WO}_{3}$ additives on sintering behaviors and magnetic properties of $\mathrm{NiCuZn}$ ferrites," Materials Science and Engineering B, vol. 117, no. 3, pp. 231-234, 2005.

[5] T. Krishnaveni, B. R. Kanth, V. S. R. Raju, and S. R. Murthy, "Fabrication of multilayer chip inductors using $\mathrm{Ni}-\mathrm{Cu}-\mathrm{Zn}$ ferrites," Journal of Alloys and Compounds, vol. 414, no. 1-2, pp. 282-286, 2006.

[6] R. C. Lecraw and R. L. Comstock, "Magnetoelastic interactions in ferromagnetic insulators," in Physical Acoustics, R. N. Thurston and A. D. Pierce, Eds., vol. 3B, chapter 4, pp. 127-199, Mason, 1965.

[7] S. R. Murthy, M. Guyot, and V. Cagan, "Ultrasonic velocity changes in Polycrystalline garnets," in Proceedings of the International Conference on Magnetism (ICM '88), pp. 134-135, Paris, France, July 1988.

[8] M. Guyot and V. Cagan, "The acoustic emission along the hysteresis loop of various ferro and ferrimagnets," Journal of Magnetism and Magnetic Materials, vol. 101, no. 1-3, pp. 256$262,1991$.

[9] L. Balamuth, "A new method for measuring elastic moduli and the variation with temperature of the principal young's modulus of rocksalt between $78^{\circ} \mathrm{K}$ and $273^{\circ} \mathrm{K}$," Physical Review, vol. 45, no. 10, pp. 715-720, 1934.

[10] S. R. Murthy, "Low temperature sintering of NiCuZn ferrite and its electrical, magnetic and elastic properties," Journal of Materials Science Letters, vol. 21, no. 8, pp. 657-660, 2002.

[11] S. R. Murthy, B. Revathi, and T. S. Rao, "Temperature and composition dependence of the elastic moduli of NiZn ferrites," Journal of the Less-Common Metals, vol. 57, no. 1, pp. 29-37, 1978.

[12] S. R. Murthy and T. S. Rao, "Effect of magnetic field and temperature on the elastic behaviour of cobalt-zinc ferrites," Journal of the Less-Common Metals, vol. 65, no. 1, pp. 19-26, 1979.

[13] Y. Kawai and T. Ogawa, "Acoustic losses in single crystals of MnZn ferrite and titanium substituted Mn-Zn ferrites," Journal of the Physical Society of Japan, vol. 45, no. 6, pp. 1830-1834, 1978.

[14] O. L. Anderson, "Derivation of wachtman's equation for the temperature dependence of elastic moduli of oxide compounds," Physical Review, vol. 144, no. 2, pp. 553-557, 1966.

[15] B. Ramaiah and S. R. Murthy, "Measurement of Elastic moduli on MnZn ferrites at several Ultrasonic frequencies," Acoustics Research Letters, vol. 21, pp. 7-10, 1997.

[16] S. R. Murthy, "Elastic behaviour and internal friction studies on thin films of NiZn ferrites," Physica Status Solidi, vol. 91, p. 519, 2002.

[17] K. Praveena, K. Sadhana, and S. R. Murthy, "Elastic behaviour of microwave hydrothermally synthesized nanocrystalline $\mathrm{Mn}_{1-x}-\mathrm{Zn}_{x}$ ferrites," Materials Research Bulletin, vol. 47, no. 4, pp. 1096-1103, 2012. 



Submit your manuscripts at http://www.hindawi.com
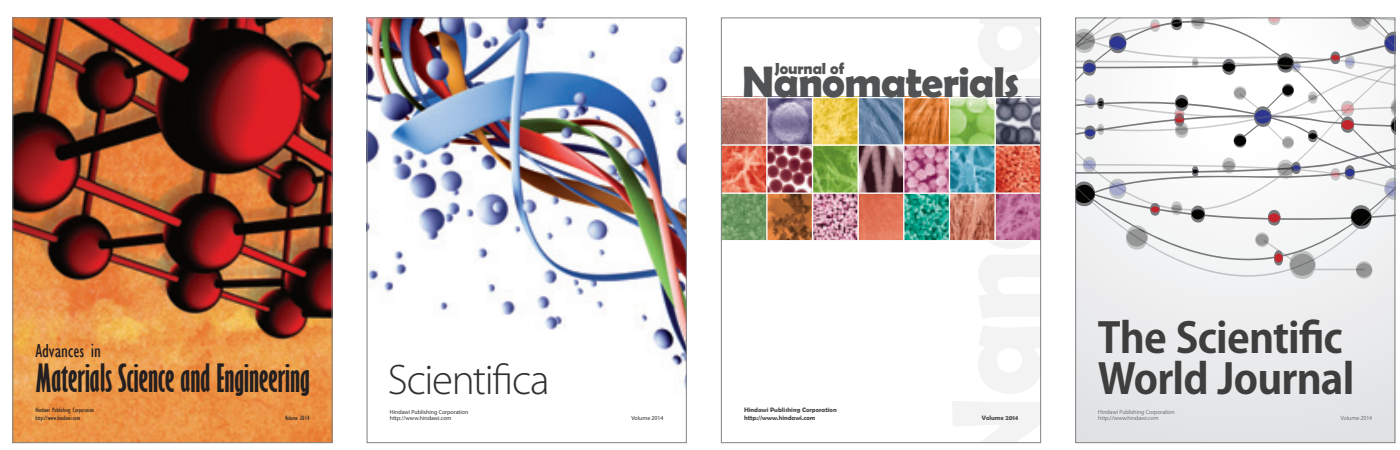

\section{The Scientific World Journal}
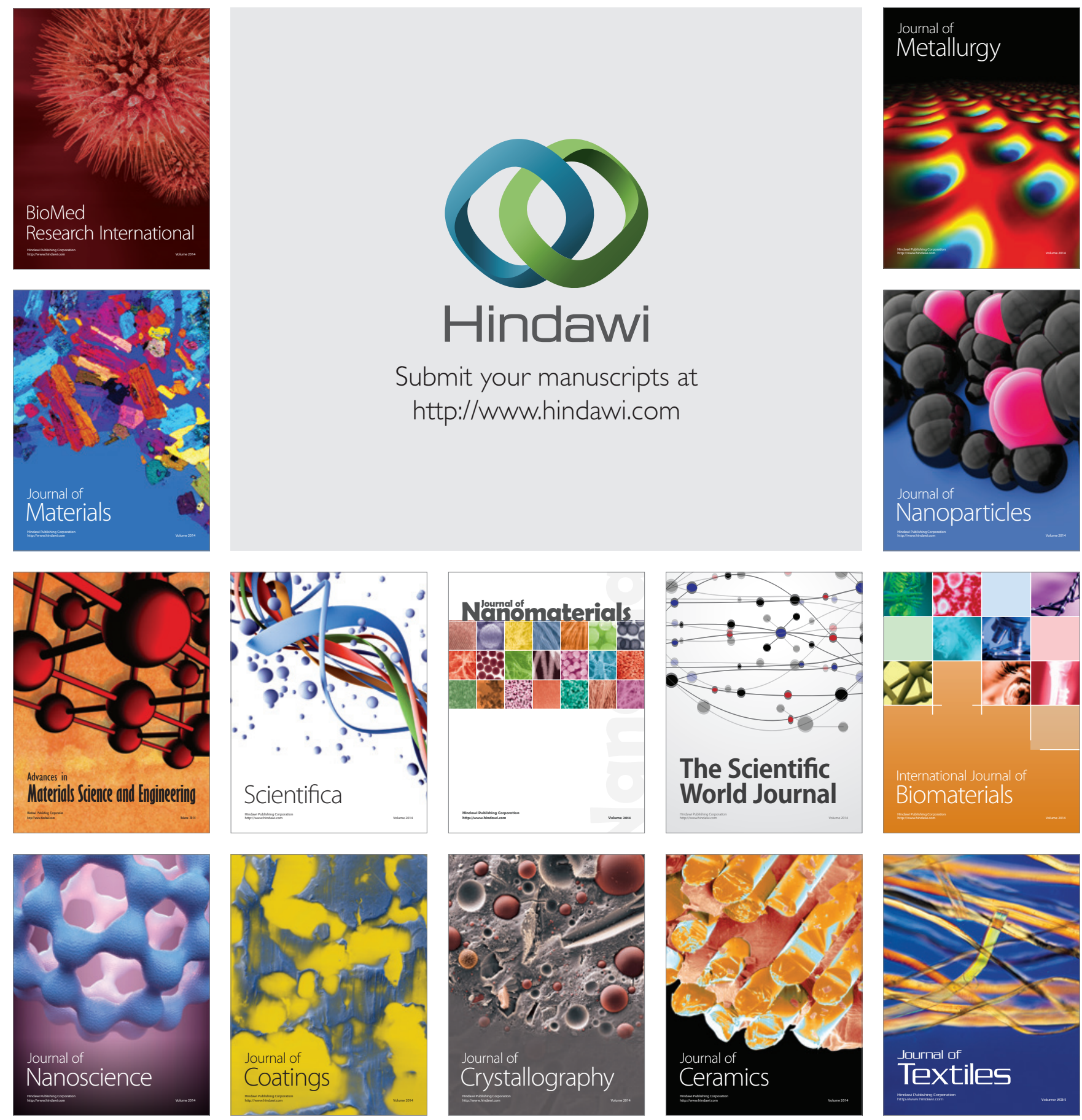